\section{SOI: $1.1 /$ TAS $\quad$ DOI: $10.15863 /$ TAS International Scientific Journal Theoretical \& Applied Science}

\section{Dmitry Lyubimov \\ $\mathrm{PhD}$ in mechanics Engineering center L \& Co, Ltd, \\ Shakhty, Russia rostexx@rambler.ru}

p-ISSN: 2308-4944 (print) e-ISSN: 2409-0085 (online)

Year: $2018 \quad$ Issue: 09 Volume: 65

Published: $18.09 .2018 \quad \underline{\text { http://T-Science.org }}$

SECTION 3. Nanotechnology. Physics
Kirill Dolgopolov

$\mathrm{PhD}$ in mechanics

Rostov State University of communication lines, Rostov-on-Don, Russia lik.lab061@gmail.com

\title{
«NONLINTAR» MODEL OF TRIBOSYSTEMS EVOLUTION
}

Abstract: A theoretical approach is presented to describe the parameters which characterize the friction interaction of solid bodies as well as the processes of selforganization at friction. Based on this approach, interconnections are established between mechanical and molecular-atomistic models of friction processes. A supposition is suggested about creation of a unified model of friction.

Key words: soliton, tribon, friction system, quantum mechanics.

Language: Russian

Citation: Lyubimov B, Dolgopolov K (2018) «NONLINTAR» MODEL OF TRIBOSYSTEMS EVOLUTION. ISJ Theoretical \& Applied Science, 09 (65): 53-58.

Soi: http://s-o-i.org/1.1/TAS-09-65-9 Doi: crossef https://dx.doi.org/10.15863/TAS.2018.09.65.9

\section{«НЕЛИНЕЙНАЯ» МОДЕЛЬ ЭВОЛЮЦИИ ТРИБОСИСТЕМ}

Аннотация: Представлен теоретический подход к описанию величин, характеризующих фрикционное взаимодействие твердых тел, а также процессов самоорганизации при трении. На основании предложенного подхода установлены взаимосвязи между механистической и молекулярноатомистической моделями процессов трения. Высказано предположение о возможности создания единой модели трения.

Ключевые слова: солитон, трибон, трибосистема, квантовая механика.

Работа выполнена при поддержке Российского научного фонда (проект №14-29-00116), организация ФГБОУ ВПО «Ростовский государственный университет путей сообщения", участник проекта Долгополов К.Н.

\section{1. Введение}

Современные тенденции к росту надежности машин при неизменном повышении энергоемкости их силовых установок и энергонапряженности процессов фрикционного взаимодействия материалов узлов трения приводят к расширению базы знаний о методах расчета теплофизических параметров конструкционных материалов, как основного критерия их трибостойкости. На сегодняшний день, в силу развития аппарата квантовохимических расчетов и химического моделирования процессов взаимодействия веществ, на первый план встает требование обеспечения заданной точности результатов вычислений при упрощении вычислительных систем и минимизации вычислительных ресурсов. Это может быть достигнуто путем применения полуэмпирических методов расчета энергии и структуры систем, достаточных для выполнения конструкторских изысканий. Облегчение такого рода расчетных задач возможно за счет формализации подхода к трению как к обмену квазичастицами, в результате чего формируется поле фрикционного взаимодействия, в плоскости которого и протекает эволюция трибосистемы.

\section{2. Движение солитонов}

Развитие представлений о микромеханизмах трения и изнашивания материалов требует применения современного аппарата физических теорий для их описания $[1,2]$. В конце прошлого века известный российский специалист по термодинамике и самоорганизации трибосистем А.А. Поляков писал: «В результате 
формирования новой области физической кинетики неравновесных процессов на сцену выходит новый фактор - квантовая структура энергетических состояний атомов и молекул. Возникает необходимость детального учета квантовой структуры вещества, всего набора энергетических термов частиц и его временной эволюции» [3].

Следует различать понятия «узел трения», «трибосопряжение» и «трибосистема» [4]. Два первых являют собой ряд технических элементов, взаимосвязанных структурно и функционально [5]. Трибосистема «возникает» в результате фрикционного взаимодействия между этими элементами. Украинский триболог Л.И. Бершадский писал, что «...определение трибосистемы...относится не столько к терминологии, сколько является принципильным вопросом строгой теоретической постановки трибологических задач. Естественное определение трибосистемы как динамической диссипативной системы состоит в том, что она представляет собой объект, в котором реализуется деградация энергии макромеханического движения(производится энтропия), т.е. включает трибовозбуждаемые объемы материалов, границу, открытую для потоков активируемых трением электричества, звука, вещества и т.П., и связи со средой (динамическую, температурную, электрохимическую и т.п.) [6]. Представление о трибосистеме оказываются тесным образом связанным с центральным понятием современной трибологии «третье тело» [7].

Генезис трибопроцессов можно свести к трем этапам, включающим рождение третьего тела, его функционирование и разрушение. Представление совокупности физико-химических изменений, происходящих в поверхностных слоях трибосопряжения в форме появления «третьего тела», представляющего собой единую физическую систему, позволяет отождествить понятие «третье тело» и «трибосистема», как продукт фрикционного взаимодействия отдельных динамически связанных элементов трибосопряжения. Концепция третьего тела в трибологии имеет ряд сходных моментов с представлениями о квазичастицах - понятия, применяемого в квантовых теориях твердого тела, конденсированных состояний вещества [8].

В модели квазичастиц процессы близкой физической природы заменялись рассмотрением одной квазичастицы, например, фонона при исследовании упругих колебаний в кристалле. В концепции «третьего тела» совокупность различных по природе взаимодействий заменяется одним мезоразмерным физическим объектом. «Третье тело», подобно виртуальным частицам, опосредовано проявляет себя в различных физических процессах, однако если виртуальные частицы - «невидимая реальность», то третье тело - это реальная абстракция, физическая модель, проявляющая себя в виде смазочных пленок, дислокационных ступенек, пластификации поверхностей трения и т.п. Третье тело -макроскопическое виртуальное квазифизическое образование, отражающее многообразие причинно-следственных связей, характеризующих процесс трения как «удивительный, не имеющий аналога природный феномен» [9].

Отождествление понятий трибосистемы и третьего тела имеет ряд важных следствий:

-во-первых, свойства трибосистемы определяются взаимодействием термодинамических потоков, о которых писал Л.И. Бершадский;

-во-вторых, в конечном счете, пусть опосредовано, но в трибосистеме заметно влияние квантовых корреляций $[8,10]$, что делает необходимым привлечение для описания фрикционного взаимодействия аппарата и понятий квантовой механики;

-в-третьих, последовательный квантовомеханический подход к описанию эволюции трибосистем, позволяет рассматривать данный процесс как проявление фундаментальных физических законов редукции волновой функции и декогеренции, что позволяет объяснить существующие парадоксы трибологии [10-14] (данный подход не противоречит принципу Оккама [15]).

Неисследованным на сегодняшний день является постдекогерентный период эволюции трибосистемы. Следуя определению трибосистемы, приведенному выше, ее характеристики обусловлены взаимодействием энергетических потоков, возникающих внутри динамически развивающихся структурных элементов трибосопряжения. Эти потоки описывались с помощью теории переноса, причиной которого в трибосопряжении являются возмущения, нарушающие термодинамическое равновесие в узле трения [16]. Для формалистического описания данных процессов используется математический аппарат нелинейной динамики $[17,18]$.

Согласно основным положением нелинейной динамики большинство подобных процессов можно рассматривать как движение особых физических объектов, получивших название «солитоны». Введение понятия «солитон» явилось важным развитием представлений о полевом строении материи, восходящем к работам А. Эйнштейна, М. Борна, Г. Ми и др. [19]. В частности, А. Эйнштейн считал, что все наблюдаемые частицы должны описываться как решение полевой модели единой 
теории поля, над которой он работал всю жизнь. Он рассматривал нелинейные полевые модели в рамках принципа общей относительности, ориентируясь на проблему объединения теории гравитации и электромагнетизма. Г. Ми и развивший его идеи М. Борн рассматривали материальные частицы как полевые образования.

Солитон, находящийся в состоянии покоя, имеет как бы две составляющие его части: статическую - частицеподобную и динамическую -периодическую во времени часть, имеющую вид стоячей волны. Подобная полевая конфигурация, переходящая из состояния покоя в движение, формально означает проведение над уравнениями, описывающими солитон, преобразований Лоренца. При этом стоячая волна превращается в бегущую [19].

В работе [18] отмечалось, что «... развитие нелинейной динамики привело к открытию классических частицеподобных объектов солитонов, описываемых лоренц-инвариантными уравнениями и имеющих непрерывный спектр скоростей $0 \leq \vartheta \leq \vartheta_{\mathrm{s}}$, где $\vartheta_{\mathrm{s}}-$ скорость звука». Движение солитонов сопровождается эффектами, связанными с конечной скоростью звука, аналогичным релятивистским эффектам специальной теории относительности. Среди этих эффектов - лоренцово сокращение ширины движущихся солитонов, изменение формы поля механических напряжений, лоренцова зависимость энергии солитона от скорости и т.п. Работы физиков-теоретиков Г.Ф. Глинского, Е. Плебанского и М. Новелло убедительно указывают на влияние нелинейных электромагнитных полей солитонов на изменение метрики пространства-времени(генерирование эффективной римановой метрики),приводящее к искривлениям световых лучей подобно тому, как это наблюдается в гравитационных полях, что сближает эффекты нелинейной электродинамики c общей теорией относительности [20,21]. Величина искажения пространства-времени находится в прямой зависимости от величины плотности энергии, в нем аккумулированной.

Наличие у солитона частицеподобной и волновой части делает его объектом дебройлевской квантовой природы, поскольку именно в солитонах наиболее ярко проявился феномен корпускулярно-волнового дуализма, легшего в основу квантовой теории материи. Поэтому подобно квантовым частицам солитоны обладают такой характеристикой, как спин, и полным набором соответствующих квантовых корреляций $[8,19]$. Применение законов квантовой механики к описанию солитонов столкнулось со сложностями, связанными с конечными размерами этих объектов. Частицы в квантовой механике характеризуются волновыми функциями, квадрат модуля которых определяет плотность вероятности обнаружения частицы в заданной точке пространства, что подразумевает представление частицы как точечного объекта. Солитон же имеет конечные размеры. Подобные конечномерные квантовые объекты, несущие на себе электрический заряд, были изучены крупными американскими физикамитеоретиками Дж. Уилером и Ч. Мизнером [22]. Они предложили рассматривать подобные частицы как области с измененной топологией пространства, обладающей значительной кривизной, что с точки зрения ОТО означает наличие в данной пространственной области значительной энергетической плотности. Частицы в интерпретации Уилера-Мизнера являются элементом измененной геометрической структуры физического пространства, рассматриваемого в форме гиперповерхностей, находящихся в четырехмерном пространствевремени.

\section{3. От солитона к трибону - путь к созданию единой теоии трения}

Математический аппарат, описывающий физическое поведение солитонов, в полной мере соответствует их дуальной квантовой природе, поэтому основные соотношения в теории солитонов имеют вид волновых уравнений Гордона или уравнения Шредингера $[8,16,17]$. В условиях трения энергетические потоки, формируемые внутри трибосистемы, можно рассматривать как движение «солитонов Хиггса» в некотором скалярном поле, описываемом полевой функцией $\Phi$ и потенциалом $V(\Phi)$ (подчиняющееся волновому уравнению вида синус-Гордона) [18,23].

Плотность импульса такого солитона определяется как $p=-\Phi \nabla \Phi$, где $\nabla$-оператор Пуассона. В модели Хиггса движение в скалярном поле можно рассматривать как одномерное $\Phi=\Phi(t ; x)$, $\quad$ т.е. описываемое уравнением [23]:

$$
\frac{\partial^{2} \Phi}{\partial t^{2}}-\frac{\partial^{2} \Phi}{\partial x^{2}}=m^{2} \phi-\lambda \phi^{2},
$$

где $m$ и $\lambda$ - постоянные интегрирования.

Если представить, что солитон перемещается в пространстве с некоторой относительной скоростью $u$ по траектории $x=x_{0}$ $+u t$, то полевую функцию $\Phi(t ; x)$ целесообразно представить в виде $f(x-u t)$, что приводит к следующей форме уравнения движения:

$$
\begin{aligned}
\frac{1}{\gamma^{2}} f^{\prime \prime} & =f-f^{3}, \\
\gamma & =\frac{1}{\sqrt{1-u^{2}}},
\end{aligned}
$$


где $u$ - отношение скорости солитона к скорости звука.

Коэффициент $\gamma$ в уравнении (2) подчеркивает лоренц-инвариантность уравнений, описывающих движение солитонов Хиггса, примером которых являются, например, дислокационные потоки. То, что движение быстрых дислокаций описывается уравнениями специальной теории относительности (СТО), было показано в середине 20 века южноамериканским физиком Дж. КульманВильсдорф, которая считала, что лоренцинвариантность в данном случае не является формальным совпадением уравнений, описывающих движение дислокаций и формул CTO, а отражает факт действия законов релятивистской физики [24]. Развитие нелинейной динамики подтвердило предположения Кульман-Вильсдорф и предопределило зарождение одной из областей трибофизики - триборелятивизма $[8,25]$. Особое влияние триборелятивистские эффекты оказывают на трибохимические процессы, что связано с зависимостями энергии и собственного времени физических объектов от скорости их движения и, соответственно - значения параметра $\gamma$ из уравнения (2). То, что такие временные изменения внутри трибосистем реально наблюдаются, подтверждено серией опытов, аналогичных оптическим экспериментам Г. Айвса [26], в которых при помощи доплеровского смещения оптического излучения было обнаружено замедление течения времени, предсказанное СТО. Эффекты, аналогичные обнаруженным Айвсом, были исследованы при прохождении лазерного пучка через фрикционный контакт [20, 27]. Вклад со стороны сил трения во временной сдвиг составляет 2 микросекунды за 1 секунду. Подобные временные отрезки оказываются больше, чем характерные времена химического взаимодействия, что подтверждает вклад триборелятивистских эффектов в течение трибохимических реакций.

«Одномерный» вид уравнения движения (1) облегчает рассмотрение термодинамической эволюции трибосистемы. При переходе к одномерному приближению солитонов Хиггса скорость изменения энтропии может быть записана в виде:

$$
\frac{d S}{d t}=\frac{1}{T^{2}} \frac{\partial}{\partial z} \Omega\left(\mathrm{Q}, \mu_{\kappa}\right)
$$

где $S$ - энтропия, $t$ - время, $T$ - температура, $Q-$ тепловой поток, $\mu_{\mathrm{\kappa}}-$ химический потенциал, $\Omega$ обобщенная термодинамическая функция.
Обобщенная термодинамическая функция может быть выражена через тепловые параметры:

$$
\Omega=\frac{\pi \lambda}{2} T^{2} z^{2}
$$

где $\lambda$ - коэффициент теплопроводности среды, $T$ - температура среды, $z$ - линейные размеры среды.

Если температуру $T$ выразить через параметры фрикционного взаимодействия в исследуемой трибосистеме с использованием закона теплопередачи Ньютона, то получим следующее выражение:

$$
T=\frac{f N \vartheta}{\pi \lambda z}
$$

приводящее к изменению выражения (4):

$$
\Omega=\frac{1}{2} \frac{f^{2} N^{2} \vartheta^{2}}{\pi \lambda}=\frac{1}{2} \frac{F_{m p}^{2} \vartheta^{2}}{\pi \lambda}=\frac{1}{2 \pi \lambda} P_{\mathrm{Tp}}^{2},
$$

где $f_{\text {тр }}$ - коэффициент трения, $N-$ нормальная нагрузка, $\vartheta-$ скорость скольжения, $F_{\text {тр }}-$ сила трения, $P_{\text {тр }}-$ мощность, развиваемая силами трения.

Выражение (5) для функции $\Omega$ делает данную величину схожей $\mathrm{c}$ диссипативной функцией. Эта связь усиливается при записи выражения (3) через мощность трения:

$$
\frac{d S}{d t}=\frac{1}{\pi \lambda z}\left(\frac{P_{m p}}{T}\right)^{2}
$$

Отношение мощности трения к температуре можно рассматривать по аналогии с классической термодинамикой как «приведенную мощность трения». Соответственно скорость изменения энтропии трибосистемы оказывается пропорциональной этой величине. Полученные соотношения, связывающие линейной зависимостью термодинамические характеристики трибосистемы и мощность трения, которая может быть измерена с достаточной точностью, возможно, открывают путь к новым способам трибомониторинга, в которых на смену силовым характеристикам фрикционного взаимодействия придут энергетические.

Анализ выражения (6), связывающего количественную характеристику воспроизведения энтропии трибосистемы с приведенной мощностью трения, предполагает ее уменьшение в процессе самоорганизации. Следствием процессов самоорганизации служит улучшение антифрикционных характеристик узла трения, происходящие благодаря формированию смазывающих пленок, экранирующих тепловые потоки на поверхности трения. 
Упрощение выражений, характеризующих кинетику изменения термодинамических параметров трибосистемы и её энергетических характеристик до вида (5) и (6), открывает дополнительные возможности в развитии квантово-механических моделей фрикционного взаимодействия. В основе квантовомеханических представлений может быть положена гипотеза, высказанная Л.И. Бершадским [20] и получившая развитие в работах $[8,30]$.

Гипотеза строится на применении к описанию фрикционного взаимодействия представления об обменных силах, введенных в квантовую физику нобелевским лауреатом Р. Фейнманом [31]. В соответствии с этой гипотезой предлагается рассматривать силу трения как результат обмена между поверхностями трибосопряжения квазичастицами «трибонами», представляющими собой кванты трения [20]. Генерирование трибонов осуществляется в контакте шероховатостей трущихся тел, а поскольку силы трения оказывают влияние сразу на обе поверхности фрикционного контакта, то можно допустить, что за единичный акт фрикционного взаимодействия двух микронеровностей рождается два одинаковых трибона - по одному на каждую поверхность. Данное предположение получило доказательство в работе [8]. В рамках развиваемых концепций трибон может рассматриваться как «элементарный солитон». Энергетические параметры, характеризующие фрикционное взаимодействие в трибосопряжении, можно представить в виде суммы физических характеристик, соответствующих отдельным трибонам, число и энергия которых в соответствии с результатами, приведенными в работах $[8,30]$, поддаются оценкам.

В работах $[8,30]$ было показано, что количество генерируемых силами трения трибонов пропорционально величине фактического давления, фактической площади контакта. В процессе фрикционного взаимодействия поверхностей трения наблюдается сглаживание шероховатостей, приводящее к уменьшению величины фактического давления, a, следовательно, к уменьшению числа трибонов, порождаемых трением. Снижение интенсивности генерирования трибонов, числом которых определяется величина мощности трения (6), характеризует завершение приработки и перехода узла трения к установившемуся режиму изнашивания [31] с минимальной скоростью прироста энтропии. В соответствии с основными принципами термодинамики данный процесс означает структурное усложнение трибосистемы как результат ее самоорганизации, по представлениям А.А. Полякова $[3,16]$.

\section{Заключение}

Подход, основанный на элементах нелинейной динамики и квантово-обменной модели, позволяет с единых позиций описывать механистический аспект представления сил трения и усложнение структуры трибосистемы, определяемое законами термодинамики. Применяемые принципы дают возможность усовершенствовать полуэмпирические методы расчета энергии, структуры, свойств трибосистемы, облегчив задачи моделирования трибопроцессов при разработке новых конструкционных материалов. Предложенный подход, являясь развитием «атомарномолекулярных» моделей трения, позволяет ближе подойти к созданию единой трибологической доктрины.

\section{References:}

1. Kolesnikov VI (2003) Teplofizicheskie protsessy $\mathrm{v}$ metallopolimernykh tribosistemakh. - M.: Nauka, 2003. - 279 p.

2. Myshkin NK, Sviridenok AI, Grigor'ev AYa (2013) Masshtabnyy faktor v tribologii // POLIKOMTRIB-2013: Tezisy dokladov mezhdunarodnoy nauchno-tekhnicheskoy konferentsii. - Gomel': IMMS NAN Belarusi, 2013. - p.8

3. Polyakov AA (1987) Dissipativnaya struktura izbiratel'nogo perenosa// Sb. «Dolgovechnost' trushchikhsya detaley mashin», vyp.2. - M.: Mashinostroenie, 1987. - p.97-106.

4. Lyubimov DN, Dolgopolov KN (2011) Fundamental'nye osnovy evolyutsii tribosistem. - Shakhty: FGBOU VPO «YuRGUES», 2011. $-95 \mathrm{p}$.

5. Chikhos Kh (1982) Sistemnyy analiz v tribonike. - M: Mir, 1982. - 348 p.

6. Bershadskiy LI (1989) O vzaimosvyazi strukturnykh mekhanizmov i dissipativnykh potokakh pri kineticheskom (nekulonovskom) 


\begin{tabular}{l|lr|ll|ll} 
& ISRA (India) & $=\mathbf{1 . 3 4 4}$ & SIS (USA) & $=\mathbf{0 . 9 1 2}$ & ICV (Poland) & $=\mathbf{6 . 6 3 0}$ \\
Impact Factor: & ISI (Dubai, UAE) $=\mathbf{0 . 8 2 9}$ & PUHЦ (Russia) $=\mathbf{0 . 1 5 6}$ & PIF (India) & $=\mathbf{1 . 9 4 0}$ \\
& GIF (Australia) & $\mathbf{0 . 5 6 4}$ & ESJI (KZ) & $=4.102$ & IBI (India) & $=\mathbf{4 . 2 6 0}$ \\
& JIF & $=\mathbf{1 . 5 0 0}$ & SJIF (Morocco) & $=2.031$ & & \\
\hline
\end{tabular}

trenii i iznose // Trenie i iznos. - 1989 (10), № 2. - p. 358-364.

7. Shvedkov EL (1979) Slovar'-spravochnik po treniyu, iznosu i smazke detaley mashin / Shvedkov E.L., Rovinskiy D.Ya., Zozulya V.D., Braun E.D. - Kiev: Naukova dumka, 1979 g. -185 p.

8. Lyubimov DN, Pinchuk LS, Dolgopolov KN (2012) Kvantovaya tribofizika. - Rostov-naDonu: Izdatel'stvo Yuzhnogo federal'nogo universiteta, 2012. - $294 \mathrm{p}$.

9. Garkunov DN (1985) Tribotekhnika. - M.: Mashinostroenie, 1985. - $424 \mathrm{p}$.

10. Lyubimov DN, Pinchuk LS, Dolgopolov KN (2013) Kvantovaya paradigma tribologii. Rostov-na-Donu: Izdatel'stvo Yuzhnogo federal'nogo universiteta, 2013. - $206 \mathrm{p}$.

11. Feygin OO (2009) Velikaya kvantovaya revolyutsiya. - M.: Eksmo, 2009. - 256 p.

12. Feygin OO (2010) Fizika nereal'nogo. - M.: Eksmo, 2010. - 272 p.

13. Arsenov OO (2011) Parallel'nye vselennye. M.: Eksmo, 2011. - 272 p.

14. Barannikov AA, Firsov AV (2009) Osnovnye kontseptsii sovremennoy fiziki. - M.: Vysshaya shkola, 2009. $-390 \mathrm{p}$.

15. Kerrol RT (2005) Entsiklopediya zabluzhdeniy: sobranie neveroyatnykh faktov, udivitel'nykh otkrytiy i opasnykh poveriy. - M.: Dialektika, 2005. - p. 78-82.

16. Lyubimov DN, Pinchuk LS, Dolgopolov KN (2011) Tribofizika. - Rostov-na-Donu: Izdatel'stvo Yuzhnogo federal'nogo universiteta, 2011. $-296 \mathrm{p}$.

17. N'yuell A (1982) Solitony $\mathrm{v}$ matematike i fizike. - M.: Mir, 1982. -121 p.

18. Musnenko AI, Manevich LI (2004) Analogi relyativistskikh effektov v klassicheskoy mekhanike // Uspekhi fizicheskikh nauk, 2004 (174), № 8. - p. 861-886.

19. Chernetskiy AA (2012) Nelineynaya elektrodinamika. - SPb.: SPBGIEU, 2012. $360 \mathrm{p}$.
20. Glinskiy GF (1980) Nelineynaya elektrodinamika i anizotropiya prostranstvavremeni // Izvestiya VUZov, seriya «Fizika». 1980, №4. - p. 52-55.

21. Plebanski J (1970) Lectures on non-linear electrodynamics. - Copenhagen: Nordita, 1970.

22. Mizner Ch, Uiler Dzh (1979) Klassicheskaya fizika kak geometriya //Sbornik statey. Al'bert Eynshteyn i teoriya gravitatsii. - M: Mir, 1979. - p. 542-557.

23. Getmanov BS (1976) Svyazannye sostoyaniya solitonov v modeli teorii polya // Pis'ma v ZhETF. - 1976 (24), № 5. - p. 323-327.

24. Fizicheskoe metallovedenie, t. 3. Pod red. R. Kana. - M.: Mir, 1968. - 430 p.

25. Lyubimov DN, Dolgopolov KN (2011) Relyativistskaya paradigma $\mathrm{v}$ tribofizike poverkhnostnykh yavleniy // Trenie i smazka v mashinakh i mekhanizmakh, 2011, №9. - p.4348.

26. Landsberg GS (2003) Optika. Izd. 6-e stereot. M.: FIZMATLIT, 2003. -848 p.

27. Lyubimov DN, Dolgopolov KN, Vershinin NK (2012) Triboaktiviruemyy doplerovskiy sdvig // Trenie i smazka v mashinakh i mekhanizmakh, 2012, №8. - p. 29-33.

28. Landau LD, Lifshits EM (2004) Teoreticheskaya fizika, t.1: Mekhanika. - M.: FIZMATLIT, 2004. $-224 \mathrm{p}$.

29. Bershadskiy LI (1992) O samoorganizatsii i kontseptsii iznosostoykosti tribosistem // Trenie i iznos, 1992(13), № 6. - p. 1076-1094.

30. Lyubimov DN, Dolgopolov KN (2012) Kvantovo-korpuskulyarnyy mekhanizm friktsionnogo vzaimodeystviya poverkhnostey treniya // Trenie i smazka v mashinakh i mekhanizmakh, 2012, №3. - p. 11-14.

31. Feynman R (2012) KED - strannaya teoriya sveta i veshchestva. - M.: Astrel', 2012. - 191 p.

32. (1995) Osnovy tribologii / Pod. red. AV Chichinadze. - M.: Nauka i tekhnika, 1995. $778 \mathrm{p}$. 